\title{
Combined PET/MR: The Real Work Has Just Started. Summary Report of the Third International Workshop on PET/MR Imaging; February 17-21, 2014, Tübingen, Germany
}

D. L. Bailey, ${ }^{1}$ G. Antoch, ${ }^{2}$ P. Bartenstein, ${ }^{3}$ H. Barthel, ${ }^{4}$ A. J. Beer, ${ }^{5}$ S. Bisdas, ${ }^{6}$ D. A. Bluemke, ${ }^{7}$ R. Boellaard, ${ }^{8}$ C. D. Claussen, ${ }^{9}$ C. Franzius, ${ }^{10}$ M. Hacker, ${ }^{11}$ H. Hricak,${ }^{12}$ C. la Fougère, ${ }^{13}$ B. Gückel, ${ }^{9}$ S. G. Nekolla, ${ }^{14}$ B. J. Pichler, ${ }^{15}$ S. Purz, ${ }^{4}$ H. H. Quick, ${ }^{16,17}$ O. Sabri, ${ }^{4}$ B. Sattler, ${ }^{4}$ J. Schäfer, ${ }^{9}$ H. Schmidt, ${ }^{9}$ J. van den Hoff, ${ }^{18}$ S. Voss, ${ }^{19}$ W. Weber, ${ }^{20}$ H. F. Wehrl, ${ }^{15}$ T. Beyer ${ }^{21}$

${ }^{1}$ Department of Nuclear Medicine, Royal North Shore Hospital, and Faculty of Health Sciences, University of Sydney, Sydney, Australia ${ }^{2}$ Institute of Diagnostic and Interventional Radiology, University Clinic Düsseldorf, Düsseldorf, Germany

${ }^{3}$ Department of Nuclear Medicine, Ludwig Maximilians University, Munich, Germany

${ }^{4}$ Department of Nuclear Medicine, Leipzig University, Leipzig, Germany

${ }^{5}$ Department of Nuclear Medicine, Ulm University, Ulm, Germany

${ }^{6}$ Department of Interventional and Diagnostic Neuroradiology, Eberhard Karls University Tübingen, Tübingen, Germany

${ }^{7}$ Radiology and Imaging Sciences, National Institutes of Health, Bethesda, MD, USA

${ }^{8}$ Department of Radiology \& Nuclear Medicine, VU University Medical Centre, Amsterdam, The Netherlands

${ }^{9}$ Department of Interventional and Diagnostic Radiology, Eberhard Karls University Tübingen, Tübingen, Germany

${ }^{10}$ Centre of Morphological and Molecular Diagnostics (ZeMoDi), MR-and PET/MRI; Centre of Nuclear Medicine and PET/CT, Bremen, Germany

${ }^{11}$ Division of Nuclear Medicine, Department of Biomedical Imaging and Image-guided Therapy, Medical University of Vienna, Vienna, Austria

${ }^{12}$ Department of Radiology, Memorial Sloan-Kettering Cancer Center, New York, NY, USA

${ }^{13}$ Department of Nuclear Medicine and Molecular Imaging, Eberhard Karls University Tübingen, Tübingen, Germany

${ }^{14}$ Clinic for Nuclear Medicine, Technical University Munich, Munich, Germany

${ }^{15}$ Werner Siemens Imaging Center, Department of Preclinical Imaging and Radiopharmacy, Eberhard Karls University Tübingen, Tübingen, Germany

${ }^{16}$ Erwin L. Hahn Institute for MR Imaging, University of Duisburg-Essen, Essen, Germany

${ }^{17}$ High Field and Hybrid MR-Imaging, University Hospital Essen, Essen, Germany

${ }^{18}$ PET Centre, FZD Research Centre Dresden, Dresden, Germany

${ }^{19}$ Department of Radiology, Boston Children's Hospital, Harvard Medical School, Boston, MA, USA

${ }^{20}$ Department of Radiology, Molecular Imaging and Therapy Service, Memorial Sloan Kettering Cancer Center, 1275 York Av, New York, NY, 10068, USA

${ }^{21}$ Center for Medical Physics and Biomedical Engineering General Hospital Vienna, Medical University Vienna, 4 L Waehringer Guertel 18-20, 1090, Vienna, Austria 


\begin{abstract}
This paper summarises the proceedings and discussions at the third annual workshop held in Tübingen, Germany, dedicated to the advancement of the technical, scientific and clinical applications of combined PET/MRI systems in humans. Two days of basic scientific and technical instructions with "hands-on" tutorials were followed by 3 days of invited presentations from active researchers in this and associated fields augmented by round-table discussions and dialogue boards with specific themes. These included the use of PET/MRI in paediatric oncology and in adult neurology, oncology and cardiology, the development of multi-parametric analyses, and efforts to standardise PET/MRI examinations to allow pooling of data for evaluating the technology. A poll taken on the final day demonstrated that over $50 \%$ of those present felt that while PET/MRI technology underwent an inevitable slump after its much-anticipated initial launch, it was now entering a period of slow, progressive development, with new key applications emerging. In particular, researchers are focusing on exploiting the complementary nature of the physiological (PET) and biochemical (MRI/MRS) data within the morphological framework (MRI) that these devices can provide. Much of the discussion was summed up on the final day when one speaker commented on the state of PET/MRI: "the real work has just started".
\end{abstract}

Key words: Hybrid imaging, Molecular imaging, PET/CT, PET/MRI, PET, MRI, Quantification, Attenuation correction, Oncology, Paediatric oncology, Neurology, Cardiology

\section{Introduction}

$\mathrm{C}$ ombined positron emission tomography and magnetic resonance imaging (PET/MRI) is a potentially unique clinical imaging modality and key research tool. Following early attempts to generate pilot data from prototype combinations of PET and MR hardware with small animal imaging in the 1990s, whole-body human PET/MRI systems were introduced in 2010, with significant impetus from the medical imaging industry. Early adopters of PET/MRI benefitted from considerable support through national research foundations. This is interesting as the leap from the initial, limited results from preclinical PET/MR imaging to the introduction of systems applicable for imaging humans was not accompanied by any significant breakthrough in combined PET/MRI-guided disease management.

The past decade has seen widespread acceptance of hybrid imaging combinations such as PET/CT and SPECT/ CT in clinical practice. However, the attitude towards PET/ MRI has been more guarded for a number of reasons, including the high initial capital cost. Some imaging experts have high hopes for PET/MRI, calling it "PET/CT without radiation", and others foresee it replacing PET/CT entirely, but the majority, including some manufacturers, would appear to favour a "wait-and-see" approach. In several situations, however, PET/MRI is recognised as having significant potential advantages for patients (e.g., reduced radiation dose in paediatric PET oncology) and, perhaps more so at this time, clinical research.

The Tübingen PET/MRI workshop was initiated in 2012 [1], the second meeting took place in early 2013 [2] and the third meeting took place a further year later in February 2014. Each meeting has brought together early adopters of the technology, manufacturers, users, critics and those simply interested in the new possibilities of PET/MRI. While the first two workshops were envisaged as a forum for both exchange of ideas among imaging experts and panellists and for providing "hands-on" sessions led by expert readers and technologists, the concept of this third workshop was modified significantly. The dedicated handson part of the workshop was condensed in 2 days with parallel interactive sessions on MRI, PET/MRI, PET/CT, clinical reporting and software solutions, with the latter being provided and supported by various vendors, who again willingly engaged in this workshop. The hands-on sessions were followed by a developmental and exploratory workshop, which was condensed in 3 days dominated by dialogue board (DB) sessions. The topics of the dialogue boards were similar to those of 2013, focussing on the following:

- Dialogue board 1: oncology

- Dialogue board 2: quantitative correction methods and standardisation

- Dialogue board 3: neurology

- Dialogue board 4: advanced PET/MRI-multi-parametric imaging

- Dialogue board 5: paediatric oncology

- Dialogue board 6: Cardiology

This year's meeting included only one round-table discussion, on the central theme of "Key applications of PET/MRI", which followed from a pilot discussion on the 
topic at the second Tübingen meeting in 2013. Now that there are over 50 PET/MRI systems in clinical use, the organisers felt certain that a broadened perspective on key applications would emerge from the audience compared with the limited experience available in 2012 and 2013.

This report summarises the key discussion points from the six dialogue boards and the round-table discussion. For each topic, we attempt to provide individual summaries and indicate major outcomes of the discussions. We attempt to highlight where progress has been achieved and to comment on areas that have not advanced as might have been anticipated. Further, we identify drawbacks in the adoption of PET/MRI in summary tables referred to as "status quo" tables. We have expanded on the general conventions to indicate progress $(\uparrow)$, steady state $(\leftrightarrow)$ and detriments $(\downarrow)$ used in earlier reports $[1,2]$ to include "suggestive of progress" ( $\nearrow$ ) and "advancement falling short of expectations" $(\searrow)$. This year, we have included the results from the 2012 and 2013 summaries for an immediate comparison of where there have been changes.

Key to dialogue board summary of recent changes in PET/MRI:

$\uparrow \quad$ Clear documented evidence of improvement in science and methodology of PET/MRI

$\nearrow$ Suggestion of improvement in methodology applied to PET/MRI but requires further investigation

$\leftrightarrow \quad$ No change but satisfactory status since previous workshop 1 year ago

$\searrow$ Little advancement in science and methodology of PET/MRI despite previous recognition of need for improvement

$\downarrow \quad$ Less clear knowledge now exists than previously, despite further developments in science and methodology of PET/MRI

\section{Dialogue Board 1: PET/MRI in Oncology}

\section{The Issues}

Combined PET/MRI has been available for clinical use since 2010. The installed base of whole-body PET/MRI systems remains small compared to that of clinical PET/CT systems. PET/MRI today is performed most frequently for oncology indications. However, most peer-reviewed publications on PET/MRI in oncology are feasibility studies based on small sample sizes and indicate either diagnostic equivalence of PET/MRI and PET/CT (i.e., no added diagnostic benefit) $[3,4]$ or minor but non-significant diagnostic benefits in selected cases [5].

The adoption of PET/MRI in oncology has been continuously challenged by the lack of protocol and workflow standardisation [1]. The dialogue board heard that such standardisation is complicated by the fact that there is a lack of consensus among MRI users and large variations in MR protocols exist. Further, direct comparison of ostensibly similar MR sequences between manufacturers is problematic. The need for integrated reporting of PET and MRI components in a single report, rather than two separate reports, was highlighted. This suggests that the imaging specialists from both disciplines should prepare the report in a single, combined manner. The panellists engaged in robust discussions on the need to perform whole-body PET/MRI examinations while acknowledging the limited flexibility of the PET/MRI systems for protocol definition and on-the-fly adjustments. Most PET/MRI in oncology today is performed with 2-deoxy-2-[ $\left[{ }^{18} \mathrm{~F}\right]$ fluoro-D-glucose $\left(\left[{ }^{18} \mathrm{~F}\right] \mathrm{FDG}\right)$ using a multi-step protocol, following a typical PET/CT protocol design (see previous meeting summaries for examples). This may not be optimal and, in fact, is being increasingly recognised as far from ideal. Workshop attendees and panellists agreed on the need to limit total acquisition and in-room time, and therefore, new approaches to PET/MRI protocols are needed. While the acquisition of PET/MRI examinations can take up to $60 \mathrm{~min}$ or more (with most of time devoted to the MR acquisition), it was felt that a maximum PET/MRI examination time of $30 \mathrm{~min}$ should be a target to make it competitive with PET/CT examinations. It was suggested that a more pragmatic approach to clinical workflow scenarios was necessary, recognising the fact that most clinical users today probably acquire more sequences than are essential or are eventually used and reported. As PET/MRI experience matures, MR sequences that have direct relevance for cancerspecific imaging should be employed in place of the "scattershot" approach of acquiring as many sequences as possible to see which produces an outcome.

Given the challenges of standardising or even harmonising MRI data acquisition, a typical conventional imaging trial was considered difficult to achieve. For this reason, panellists and attendees suggested an alternative of creating a PET/MRI registry for pooling data acquired at multiple centres, similar to that which was developed for PET/CT in the National Oncologic PET Registry (NOPR) (see, for example, [6-9]). The aim of the registry would be to gather a sufficiently large amount of clinical data in specific clinical areas to help derive diagnostic accuracy measures (e.g., sensitivity, specificity, accuracy) and outcome data and to document measures of change in patient management, as in the NOPR project [6-9]. This could be further improved to supply earlier results if centralised reading was done at the core registry.

\section{Recent Advances or Achievements}

Numerous studies such as Aznar et al. and Bezrukov et al. $[10,11]$ have demonstrated the effect of omitting PET photon attenuation due to the bone during MR-derived attenuation correction (MR-AC) in PET. No major methodological progress applicable to whole-body oncology imaging has been reported in the past year. From a clinical perspective, only incremental progress has been demonstrated in head and neck cancers [12], breast cancer [13], paediatric oncology imaging $[14,15]$, peritoneal carcinomatosis [16], glioma [17] and prostate cancer [18-22]. 


\section{New Evidence That Has Been Reported (Table 1)}

Several groups have proposed refinements to whole-body imaging protocols $[13,23]$. The panellists disagreed on the clinical usefulness and clinical need for whole-body diffusion-weighted imaging (DWI) sequences in the context of PET/MRI imaging. DWI and $\left[{ }^{18}\right.$ F]FDG-PET imaging were considered "complementary", while acknowledging the fact that standardised approaches to reporting and analysing these images were still missing. At this point in time, diffusion-weighted images were considered superfluous if co-registered $\left[{ }^{18} \mathrm{~F}\right] \mathrm{FDG}$ images were available. However, DWI could replace $\left[{ }^{18} \mathrm{~F}\right]$ FDG imaging from a functional perspective, as they are thought to provide similar information, thus permitting an alternative PET radiopharmceutical to be used (e.g., $3^{\prime}-\left[{ }^{18} \mathrm{~F}\right]$ fluoro-3'-deoxythymidine $\left(\left[{ }^{18} \mathrm{~F}\right] \mathrm{FLT}\right), 6-[18 \mathrm{~F}]$ fluoro-L-DOPA $\left.\left(\left[{ }^{18} \mathrm{~F}\right] \mathrm{DOPA}\right)\right)$. It has even been hinted in some areas of oncology where MR imaging plays an important role (e.g., brain tumours, prostate cancers, pancreatic and liver cancers) that $\left[{ }^{18} \mathrm{~F}\right]$ FDG imaging may not be the PET agent-of-choice for a complementary investigation. This could eventually make the decision of whether to use PET/CT or PET/MRI easier; if the cancer is $\left[{ }^{18} \mathrm{~F}\right] \mathrm{FDG}$-avid, then the study is likely best performed with $\mathrm{PET} / \mathrm{CT}$, whereas other radiopharmaceuticals may be a better choice for PET/MRI.

\section{Future Challenges}

A major challenge as stated by one panellist was that "technical innovations appear to outpace regulatory processes and legislation". This is demonstrated by the fact that, while multi-modality imaging was introduced into clinical use over a decade ago, reimbursement is still often nonexistent and varies widely between countries. Users should focus on developing unique clinical applications for PET/ MRI and its use to probe human physiology and pathophysiology in cardiology, neurology and oncology. In particular, evidence needs to be gathered to demonstrate that PET/MRI affects patient-relevant outcomes when compared to separate MRI and PET/CT; this could be achieved through a registry as addressed in the round-table discussion on key applications.

While the training schemes for expert readers of dualmodality imaging remain a matter of debate and no consensus has been reached yet, it was agreed that the most appropriate way to accelerate the advancement of PET/MRI technology will be through team efforts of imaging experts with different backgrounds so that they will teach and learn from each other for the benefit of patients.

Questions raised by the dialogue board include the following:

- Is PET/MRI equivalent to separate PET/CT and MRI and what is the advantage of simultaneous PET and MR imaging?

- How can the advantages be assessed from a health care perspective? How does each modality influence relevant clinical endpoints?

- Is PET/MRI currently more suitable for use as a research tool than for routine clinical practice?

\section{Dialogue Board 2: Quantitative Correction Methods and Standardisation}

\section{The Issues}

The ability of PET to quantify physiological and metabolic pathways non-invasively needs to be maintained in combined PET/MR. This requires clinically viable and validated approaches for MR-based correction of attenuation and scattered radiation. All approaches need to account for the presence of the scanning bed, positioning aids and MR radiofrequency (RF) coils employed during the combined examination. Inaccuracies in generating correct attenuation factors from the MR-based approaches remain one of the weakest points in the technology of PET/MRI today. Anything less than achieving accuracy in attenuation correction factors equivalent to CT-based methods will be seen as sub-optimal and a regressive step.

\section{Attenuation Correction}

Standard PET/MRI AC is based on MR imaging using preselected sequences followed by image segmentation that classifies the resulting attenuation map into three or four classes of tissues (e.g., background, soft tissue, fat, lung) each with a single assumed attenuation value [24]. This approach is reasonably accurate for average size patients but fails in very large patients (truncation) or patients with dense or metallic prostheses (susceptibility artefacts). In addition, the reproducibility of standard MR-AC with Dixon fat/ water sequences may occasionally be hampered by inverted tissue

Table 1. Status quo DB1: PET/MRI in oncology

Diagnostic quality of PET in PET/MRI equivalent to PET quality in PET/CT Resolving quantitative bias from MR-AC

Clinical data available on diagnostic accuracy of PET (/MRI) in oncology PET/MRI protocol standardisation

Definition of key clinical applications

\begin{tabular}{lll}
2012 & 2013 & 2014 \\
\hline$\leftrightarrow$ & $\leftrightarrow$ & $\nearrow$ \\
$\searrow$ & $\leftrightarrow$ & $\leftrightarrow$ \\
$\leftrightarrow$ & $\leftrightarrow$ & $\nearrow$ \\
$\downarrow$ & $\leftrightarrow$ & $\nearrow$ \\
$\leftrightarrow$ & $\leftrightarrow$ & $\nearrow$
\end{tabular}


classifications (e.g., fat/water swaps) and the resulting incorrect tissue class and attenuation values assigned in the resulting attenuation maps. In addition, the delineation of lungs on MR images occasionally fails, and typically, only uniform attenuation values for the lung tissue are assigned. Furthermore, all MR-based attenuation maps are currently being presented without the bone being included, thus leading to a local underestimation of the attenuation coefficients [2, 25].

\section{Motion Correction and Co-registration Errors}

Local misalignment of PET and MR image information can be limited a priori through more rigid patient fixation (e.g., rigid head RF coil) compared to PET/CT. However, residual motion-induced misalignment of structures in the abdomen and thorax remains an issue. MR does, however, offer the possibility to use certain acquisition techniques (e.g., "navigator" sequences or fast, timeresolved 3D data acquisition of body volumes) to measure motion and the displacement and to allow correction for this during PET data reconstruction. This represents a potential major development for PET achieved by using complementary information provided by MR that PET/CT is unable to provide.

\section{PET Image Reconstruction}

All PET/MRI systems employ iterative reconstruction algorithms for the PET emission data, similar to those used in PET/CT. Point spread function (PSF) modelling is increasingly being integrated into commercially available reconstruction software, thus helping to improve noise characteristics and spatial resolution of the final emission images. However, the inclusion of PSF modelling into the reconstruction process may induce ringing ("Gibbs") artefacts (i.e., overshoots at object boundaries) that can lead to substantial overestimates of true standardised uptake values (SUVs) of up to about $40 \%$ in small lesions [26]. While this problem is not specific to $\mathrm{PET} / \mathrm{MR}$, the problem is one that needs to be investigated further.

\section{Quantification}

Several reports from single-injection, dual-imaging studies demonstrated discrepancies in SUVs between PET (/CT) and PET (/MR) data that require further clarification. This is not surprising as differences could arise from a number of causes such as the following:

- Physiologically different uptake at the two imaging time points (highly likely)

- Variations of the PET SUV from inaccurate attenuation correction from MR-AC compared to the CT-AC, including lacking spatial information about placement of flexible, nonstationary MR RF coils (likely)

- Differences between reconstruction algorithms between vendors (likely) and between PET/CT and PET/MR systems of the same vendor (confirmed)

- Inaccurate calibration of the PET system (possible)

These differences are related only in part to the known shortcomings of MR-AC. In addition, discrepancies were reported for different commercial systems.

\section{Recent Advances or Achievements}

\section{Attenuation Correction}

Since the last workshop, continuous progress has been made in the understanding and correction of artefacts and bias arising from truncation and MR surface RF coils. Schramm et al. proposed a straightforward compensation technique for truncation artefacts, whereby the missing attenuation information is estimated from a combination of the truncated attenuation map and the body outline of the PET images without attenuation correction [25]. Nuyts and colleagues proposed a maximum a posteriori algorithm for estimating the missing part of the attenuation map from the PET emission data [27]. This method is based on prior work on an algorithm to estimate attenuation data from available emission data only [28-30]. An alternative method was proposed by Blumhagen et al. who suggested optimising the imaging read-out gradient field by compensating for local $B_{0}$ magnetic field inhomogeneities and gradient non-linearities, thereby extending the MR field-of-view and, thus, the useful MR image information prior to deriving the MR-based attenuation correction information [31]. Kartmann et al. describe a method to align CT-based templates of flexible MR surface RF coils through the use of fiducial markers seen on both CT and MRI, thereby accounting for the additional attenuation effects from ancillary objects [32]. Bezrukov et al. developed a modified atlas-based attenuation correction for whole-body imaging [11]. By predicting the bone tissue in selected body regions, the method improved in calculation efficiency and robustness. Additionally, an atlas-based detection of artefacts was proposed.

Rezeai and Defrise et al. have shown that if the PET data component is acquired using time-of-flight acquisition, the attenuation factors may be derived from the emission data alone [29, 30, $33,34]$. This remarkable discovery is extremely promising and may prove ultimately to be the method of choice for AC in PET/MR.

\section{Motion Correction and Co-registration Errors}

Wurslin et al. described a pilot approach towards non-rigid MRbased motion correction for PET/MRI of the abdomen and thorax [35]. They demonstrated an increase in SUV of lesions of $30 \%$ compared to the non-corrected PET data, while the SNR improved by about $30 \%$ compared to a standard end-expiratory gating.

\section{Image Reconstruction}

As mentioned previously, the increasing awareness of Gibbs artefacts from the combination of iterative reconstruction methods and PSF modelling has supported the development of alternative reconstruction algorithms [36]. These authors report on the use of a volume-ofintersection, or tube-of-response (TOR), approach instead of the standard line-of-response approach to data storage and reconstruction. This TOR-based reconstruction was implemented on a Philips Ingenuity PET/MRI system and has been shown to achieve spatial resolution and noise levels equivalent to those obtained by conventional PSF modelling while suppressing Gibbs-based overshoots in small object boundaries.

\section{Quantification}

Despite prior focus on the absolute need to preserve the quantitative accuracy of the PET measurements [37], no major progress leading to increased quantitative accuracy was reported. 

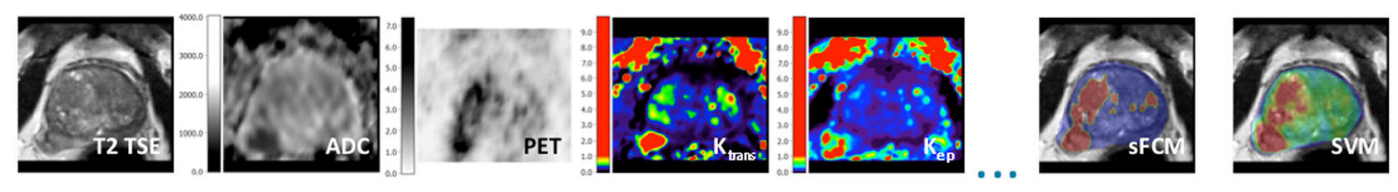

\section{Multi-parametric Imaging}

\section{Complementary information}
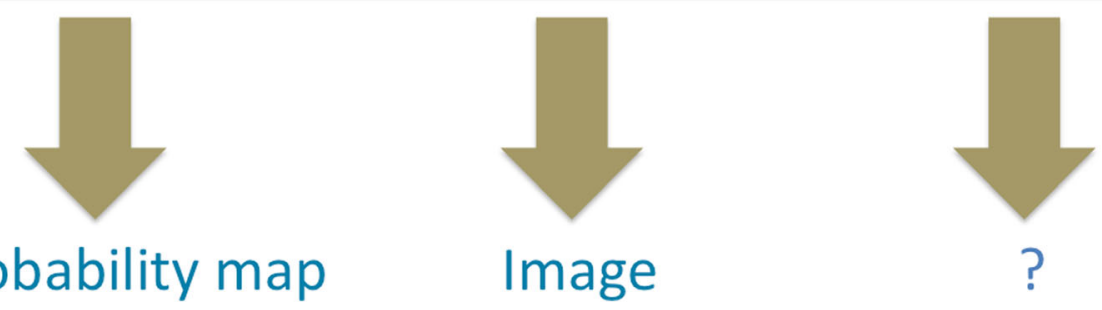

Fig. 1 Illustration of the concept of multi-parametric imaging providing multiple layers of information that can be combined (or extracted) into probability maps (based on machine learning), image-based representations of selected information or other information. sFCM spatially constrained fuzzy c-means, SVM support vector machine (with support of Dr. Gatidis, Tübingen).

In addition to engaging in the topical discussions, panellists presented new approaches for phantom-based quality control (QC) procedures for PET/MR, acknowledging the fact that there would be no image quantification without periodic and appropriate quality assurance of the systems. On this point, the use of standardised image quality phantoms filled with water-based solutions was discussed in an effort to obtain reproducible and accurate MR-based attenuation maps (Fig. 1) [38]. MRbased attenuation maps of standard phantoms, however, do not account for plastic phantom housings and, thus, lead to systematic bias in PET quantification during phantom measurements. For standard phantom tests, the use of CT-based attenuation templates of the fluid-filled standards was suggested [39]. In general, quality control requirements of PET/MRI should be similar to those for PET/CT. In particular, multicentre QC programs are hampered by the lack of dedicated QC protocols and phantom MR-AC methods, in addition to differing implementation of MR-AC methods. Initial pilot data were presented at the workshop addressing both phantom-based QC procedures and a novel approach towards volunteer-based and intra-individual verification of the accuracy of attenuation maps derived from MR on different PET/MRI systems.

\section{New Evidence That Has Been Reported (Table 2)}

The first data were presented on alternatives to PET-derived SUV approaches, reducing the dependency on valid system calibration as well as any non-linear correlation between SUV and metabolic rate, by using the PET arterial blood pool signal derived from large vessels on MRI in the FOV as an internal reference and thus replacing SUVs with target-to-blood ratios for quantitative evaluation [40]. This should be acceptable as long as relative regional contrast is preserved, which is a much weaker requirement regarding $\mathrm{AC}$ than demanding quantitatively correct absolute tissue concentrations of the radionuclides $(\mathrm{kBq} /$ $\mathrm{ml}$ ) cross-calibrated to another piece of equipment such as the dose calibrator or automated injector. Furthermore, dual-time-point measurements might provide additional advantages for quantitative PET/ MRI [41]. Panellists agreed that the capability to derive absolute tracer concentrations from the PET data was the ultimate goal of PET/MRI.

\section{Future Challenges}

Single-injection, dual-imaging studies involving PET/CT and PET/ MRI comparisons should be discontinued and replaced by singleinjection, single PET/MRI studies. This is mainly because pathologies that are suited to characterisation and response assessment with $\left[{ }^{18} \mathrm{~F}\right] \mathrm{FDG}$ are already well-suited to $\mathrm{CT}$ definition, while pathologies where MRI has an advantage may be better matched with other PET tracers, as mentioned previously.

Future challenges include the need to refine MR-AC methods (such as by increasing the repeatability of the MR-based attenuation maps) and the exploration of potential improvements of PET quantification through the use of dynamic MR image information for MR-based quantification of PET (e.g., from an image-derived input function (IDIF)). Finally, the panellists agreed on the need to introduce and adopt guidelines for quality control, standardisations and general imaging procedures.

Table 2. Status quo DB 2: Quantitative correction methods and standardisation

\begin{tabular}{|c|c|c|c|}
\hline & 2012 & 2013 & 2014 \\
\hline Critical evaluation of MR-AC methods & $\nearrow$ & $\uparrow$ & $\uparrow$ \\
\hline Validation of MR-based motion correction & $\leftrightarrow$ & $\leftrightarrow$ & $\nearrow$ \\
\hline Agreement on acceptable lower limits of quantitative accuracy of PET following MR-AC & $\searrow$ & $\searrow$ & $\leftrightarrow$ \\
\hline Clinical introduction of advanced, MR-based quantitative parameters (e.g., IDIF) & $\downarrow$ & $\searrow$ & $\searrow$ \\
\hline
\end{tabular}




\section{Dialogue Board 3: PET/MRI in Neurology}

\section{The Issues}

Neurology, neuro-oncology and neuroscience are thought to be potential key applications for PET/MRI in clinical practice and for research, since both modalities provide complementary morphological, functional, (patho)-physiological and molecular information regarding the human brain [42]. The main advantage of integrated PET/MRI systems for neurology applications is seen in the simultaneity of data acquisition, which allows both temporal and spatial crosscorrelation and potential cross-validation of PET and MR measurements [43-45]. Simultaneous PET/MRI may provide a better understanding of functional (BOLD-fMRI), haemodynamic (arterial spin labelling (ASL), proton-weighted imaging (PWI), $\left[{ }^{15} \mathrm{O}\right] \mathrm{H}_{2} \mathrm{O}$ PET) and metabolic (dynamic PET with different radiotracers, MR spectroscopy) interaction during brain activation as well as in various neurological disorders [46]. Furthermore, it is thought that the use of MRI data can help improve PET quantification through, for example, motion correction. To balance these issues, MRbased attenuation correction in the brain remains problematic especially around dense areas of the skull.

\section{Recent Advances or Achievements}

Several technical improvements have been achieved in recent times. Most are related to improving accuracy of the PET (/MR) reconstruction through the use of improved MRAC methods [47, 48]. Further, combining segmentation and atlas-based algorithms by incorporating dual-echo ultrashort echo time (DUTE) and T1w-MRI data with a probabilitybased atlas yields attenuation maps similar to those from CT-based approaches [49].

\section{New Evidence That Has Been Reported (Table 3)}

Simultaneous PET/MRI in acute stroke has revealed an insufficient estimation of the penumbra volume by perfusion-weighted MRI as compared to the gold standard approach to non-invasively measuring cerebral blood flow, $\left[{ }^{15} \mathrm{O}_{\mathrm{H}} \mathrm{O}\right.$ PET.

Dual time-point amyloid PET/MRI enables an integrated approach to imaging in dementia. More specifically, early post-injection amyloid imaging can be used as a surrogate of neuronal integrity, while delayed amyloid imaging provides information about amyloid load in the brain; anatomical MRI examines morphological changes such as atrophy, vascular lesions and space-occupying lesions. Alternatively, MR-based ASL might be useful to provide information regarding neuronal injury when acquired in parallel with the amyloid PET signal $[44,50]$.

Simultaneous PET/MRI acquisition does not necessarily come with full simultaneity of PET and MR, due to inherent differences in measurement algorithms and the temporal resolution of both imaging methods (e.g., continuous measurement for tracer kinetics vs. repetitive block design for fMRI). Emerging evidence for imaging brain tumours by means of integrated PET/MRI with amino acid analogues supports the notion that this approach has great potential to improve diagnostic accuracy, for instance, in early detection [51], in imageguided biopsy planning [14] and in therapy monitoring [52]. Further, multi-parametric image data analysis and interpretation might in the future improve patient-tailored treatment decisions.

Simultaneous PET/MRI also provides early evidence for detecting changes in neurotransmission and neuronal activity and brain networks at the same time [46, 53], which may help improve the development of new drugs. This accounts, for instance, for the effect of nicotine on cognition in Alzheimer's disease, which may be related to both a specific action on nicotinic receptors as well as acting on brain networks in general $[54,55]$.

\section{Future Challenges}

To render combined PET/MRI fully acceptable for clinical and research applications in neurology, MR-AC methods need to be implemented that account for bone attenuation and potential image distortions (e.g., susceptibility artefacts in MR-based attenuation maps). This is likely to be extremely important for PET/MRI of Alzheimer's disease, for which clinically approved radiotracers are available today. Given the subtle differences in radiotracer activity in Alzheimer's disease, the PET/MRI must accurately reflect the tracer distribution to allow confident diagnosis. Upcoming clinical studies should include the crossvalidation of MR- and PET-based physiological measurements and the continued development of IDIF for radiotracer modelling.

Table 3. Status quo DB3: PET/MRI in neurology

\begin{tabular}{|c|c|c|c|}
\hline & 2012 & 2013 & 2014 \\
\hline Improved understanding of brain physiology and function through the use of combined PET/MRI & $\leftrightarrow$ & $\nearrow$ & $\nearrow$ \\
\hline Methodological progress for improved quantification of PET/MRI neurological examinations (AC, IDIF, SUV) & $\leftrightarrow$ & $\leftrightarrow$ & $\nearrow$ \\
\hline MR-based motion correction for routine clinical use & $\downarrow$ & $\searrow$ & $\leftrightarrow$ \\
\hline
\end{tabular}




\section{Dialogue Board 4: Advanced PET/ MRI-Multi-Parametric Imaging}

\section{The Issues}

Most of the installed clinical and custom-built preclinical PET/ MRI systems support simultaneous PET and MRI data acquisition, thus enabling the contemporaneous collection of complex and temporally variable biological parameters. These parameters include the MR-based apparent diffusion coefficient (ADC) - a measure of intracellular diffusion that reveals information for oncology about tumour viability, necrosis or apoptosis - along with complementary $\left[{ }^{18} \mathrm{~F}\right] \mathrm{FDG}$ uptake as a measure of glucose metabolism. While ADC and $\left[{ }^{18} \mathrm{~F}\right] \mathrm{FDG}$ uptake values can now be measured simultaneously, it is not clear how these parameters match under conditions such as tumour progression, posttherapeutic changes or treatment response. Understanding how MRI and PET parameters match is perhaps even more challenging when considering non- $\left[{ }^{18} \mathrm{~F}\right] \mathrm{FDG}$ tracers that target other characteristics of cancer cells such as hypoxia (e.g., $\left[{ }^{18} \mathrm{~F}\right]$ fluoromisonidazole $\left(\left[{ }^{18} \mathrm{~F}\right] \mathrm{FMISO}\right),\left[{ }^{18} \mathrm{~F}\right]$ fluoroazomycin-arabinoside $\left.\left(\left[{ }^{18} \mathrm{~F}\right] \mathrm{FAZA}\right)\right)$, proliferation $\left(\right.$ e.g., $\left.\left[{ }^{18} \mathrm{~F}\right] \mathrm{FLT}\right)$ or amino acid metabolism (e.g., $\left[{ }^{18} \mathrm{~F}\right]$ fluoroethyltyrosine (FET)). In neurological stimulus experiments, the temporal alignment of PET and MRI data becomes even more critical, since every read-out must be linked to a specific stimulus, which may not necessarily be reproducible if imaging is done separately; this makes synchronous acquisition of PET and MR data a priori a requirement.

One challenge in dealing with multi-parametric data sets is that the large amount of complex information provided generally makes it difficult to take into account all measured parameters and extract core information. Utilising only conventional image information does not exploit the full potential of integrated PET/MRI. While in current clinical approaches that very basic image quantification (e.g., SUV) dominates, clinical research applications require the exploration of alternative and perhaps more complex measures, leading to the analysis of multi-parametric data, which is not yet fully supported by the available software platforms provided with today's standard PET/ MRI hardware (Fig. 2). One possible solution is the application of automated classification algorithms, which support objective, reproducible and comprehensive data analysis. These algorithms make use of unsupervised [56] or supervised [57, 58] classification.

\section{Recent Advances or Achievements}

The preclinical field pioneered the complex analysis of multi-modality PET/MRI data with enormous potential for clinical translation. Preclinical PET/MRI studies in neurooncology showed the additional value of co-acquiring in vivo $\mathrm{MR}$ spectroscopic data, $\mathrm{T} 2 \mathrm{~W}$ images and $\mathrm{PET}$ measurements. Using a glioma mouse model, chemical shift imaging to detect endogenous choline depicted gliosis, while $\left[{ }^{11} \mathrm{C}\right]$ choline PET showed the viable tumour core [59]. Thus, endogenous choline and $\left[{ }^{11} \mathrm{C}\right]$ choline uptake in PET provided complementary, differing, but valuable information about the tumour microenvironment. Advanced simultaneous PET/MRI cerebral activation studies applying BOLD-fMRI and $\left[{ }^{18} \mathrm{~F}\right]$ FDG-PET have revealed complementary brain networks as a response to whisker stimulation in the rat [60].

Few publications using classification algorithms in analysis of PET/MRI data already exist. Schmidt et al. showed that Gaussian distribution models on $\left[{ }^{18} \mathrm{~F}\right] \mathrm{FDG}-\mathrm{PET}$ and ADC can be used to separate different tissue regions within tumours of bronchial carcinoma patients [61]. Dukart et al. used a support vector machine classification algorithm to detect and differentiate Alzheimer's disease and fronto-temporal lobar degeneration from $\left[{ }^{18} \mathrm{~F}\right] \mathrm{FDG}-$ PET and MR images [62].

\section{New Evidence That Has Been Reported (Table 4)}

New evidence based on larger cohorts was not presented. Panellists discussed the promise of anecdotal pilot data from prostate cancer patients undergoing multi-parametric PET/ MR imaging (T2-weighted MRI, ADC, kinetic parameters
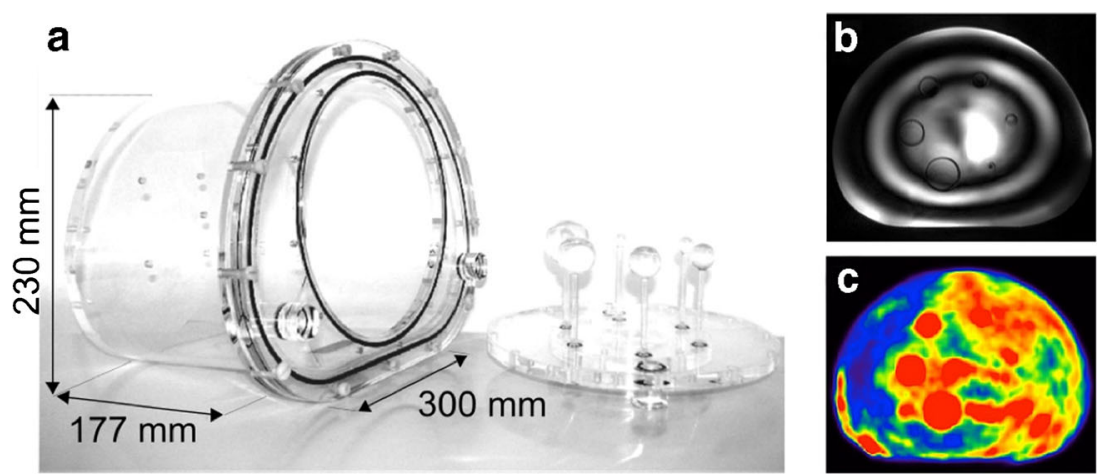

Fig. 2 The IEC 61675-1 standard body-phantom: a For imaging, the phantom can be filled with fluid and tracer. Examples of inhomogeneous MR excitation in water (b) and inhomogeneous PET-tracer distribution in oil-based substances (c) in the described phantom are shown in the images on the right. 
Table 4. Status quo DB4: Advanced PET/MRI and multi-parametric imaging

\begin{tabular}{|c|c|c|c|}
\hline & 2012 & 2013 & 2014 \\
\hline Fully integrated PET/MRI exclusively offers the largest variety of multi-parametric biomarkers & $\leftrightarrow$ & $\nearrow$ & $\uparrow$ \\
\hline Validation of advanced multi-parametric biomarkers in clinical research (beyond "image fusion") & $\searrow$ & $\leftrightarrow$ & $\nearrow$ \\
\hline Contributions of small animal imaging to the understanding of multi-parametric biomarkers & $\leftrightarrow$ & $\nearrow$ & $\uparrow$ \\
\hline
\end{tabular}

from dynamic contrast-enhanced MRI and $\left[{ }^{11} \mathrm{C}\right]$ choline PET) hinting at high accuracy values.

\section{Future Challenges}

Quantitative MR parameters are often inconsistent and vary significantly between studies; these variations are caused mainly by variability in protocols, analysis methods and non-standardised workflows. In addition, PET tracer uptake is not entirely dependent on the availability of specific transporters or receptors but instead depends also on physiological parameters such as blood flow, oxygenation or, in preclinical small animal research, anaesthesia. Thus, the inter-study variability of PET and MR data mandates reliable and standardised imaging protocols. Furthermore, acquisition protocols and data analysis tools (in-house or vendor-specific) need to be evaluated or established. The benefit of multi-parametric imaging data and its analysis using classification algorithms for detection of diseases and assessment of therapy response needs to be addressed in the future.

\section{Dialogue Board 5: PET/MRI in Paediatric Oncology}

\section{The Issues}

Paediatric oncology continues to be a focus for expanding the application of PET/MRI, with an opportunity to take advantage of the benefits of reduced radiation exposure of the paediatric patients [15] and the potential gains afforded by a single integrated PET/MRI examination. The European paediatric centres to date have been more successful at implementing PET/MRI protocols than their North American counterparts, but this is a trend that will likely shift in favour of more universal utilisation of PET/MRI in the majority of larger medical centres. However, there is general consensus on the need to produce high-quality evidence to support and expand the utilisation of PET imaging in paediatric oncology, where the majority of cases to date are still primarily the lymphoma and Ewing sarcoma.

\section{Recent Advances or Achievements}

Over the past year, representatives of three major German treatment centres reported that they had moved to performing PET/MRI almost exclusively for children with cancer, unless there are specific contraindications to doing so [63]. Following the presentation of detailed clinical perspectives, the panellists agreed on standard protocol considerations when imaging children and adolescents with combined PET/MRI (Fig. 3).

For staging of Hodgkin's lymphoma (HL), the panel felt that PET/MRI can now be used with confidence to evaluate the sites of disease and is superior to PET/CT for detecting and characterising spleen and bone marrow involvement. The use of contrast for the MRI examination is essential, and dynamic contrast sequences can further enhance the accuracy of lesion detection [64]. For restaging of HL in adolescents, DWI complements the $\left[{ }^{18} \mathrm{~F}\right] \mathrm{FDG}-\mathrm{PET}$ findings in predicting treatment response, suggesting that PET/MRI combined with DWI could provide added value [65]. Furthermore, the surveillance of lymphoma patients undergoing chemotherapy may benefit from the incorporation of chemical shift MR into the protocol to aid in distinguishing residual lymphoma from thymic rebound [66].

All panellists agreed that PET/MRI can effectively replace $\mathrm{PET} / \mathrm{CT}$ for the majority of lymphoma patients, including patients who underwent a PET/CT for initial staging. In addition, lymphoma patients receiving a restaging PET/MRI examination may not require repeat $\mathrm{CT}$ imaging of the thorax unless the MR images are considered unsuitable for excluding lung nodules.

Imaging of other tumour types, e.g., soft tissue sarcoma, germ cell tumours and thyroid cancer, will likely follow the sarcoma protocol for initial staging and response assessment, with tumour-specific sequences, contrast agents, and imaging planes as appropriate (Fig. 3).

\section{New Evidence That Has Been Reported (Table 5)}

Panellists from Tübingen reported on a local study of 20 examinations of 18 patients undergoing PET/CT and PET/MRI on the same day, which showed that MRI provided additional value in characterising soft tissue lesions, and offered significant radiation dose reduction as compared to CT [67]. There was evidence suggesting that clinically significant lung nodules were visualised by both PET/MRI and PET/CT, although CT remains superior for detecting small lung nodules [63]. The panellists further emphasised the role of PET/MRI in neurofibromatosis-1 (NF-1) patients being screened for development of malignant peripheral nerve sheath tumours (MPNST). However, there was no consensus on how frequently the combined PET/MRI imaging surveillance scan 
Disease specific considerations

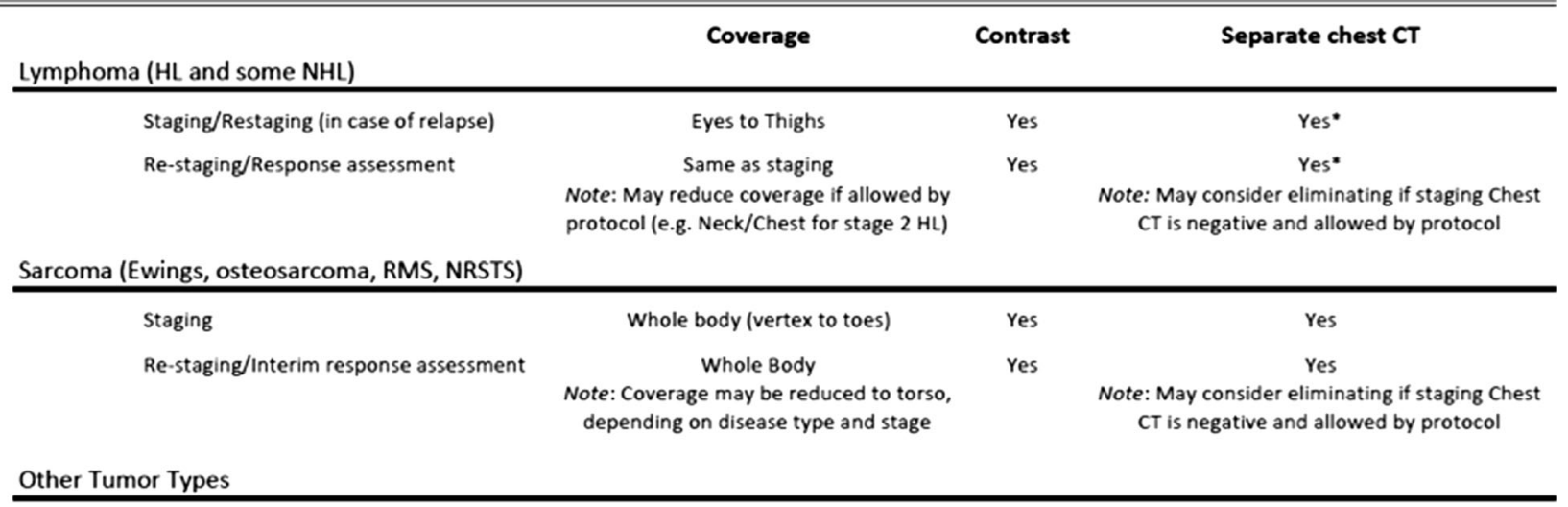

Likely to follow the sarcoma algorithm for initial staging, with tumor specific sequences, contrast agents, and imaging planes as appropriate for the tumor type.

Core Whole Body Diagnostic MR sequences for Pediatric Oncology PET/MR

\begin{tabular}{|c|c|c|}
\hline Purpose & Coverage area & MR Sequence type \\
\hline \multicolumn{3}{|l|}{ Required } \\
\hline $\begin{array}{c}\text { Attenuation } \\
\text { Correction }\end{array}$ & Whole body & Coronal T1 30 VIBE Dixon \\
\hline Recommended & & \\
\hline
\end{tabular}

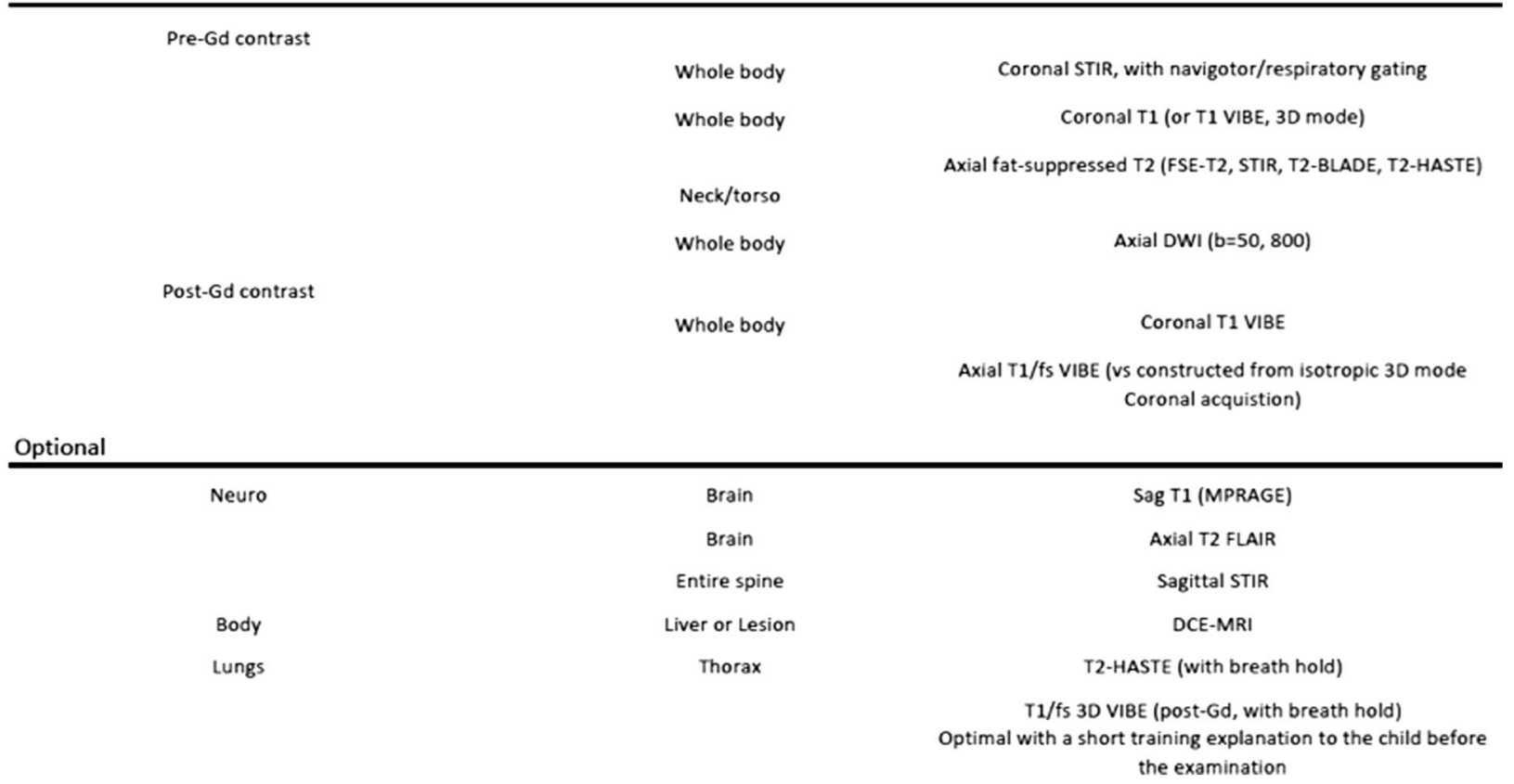

Fig. 3 Current recommended applications of PET/MRI in paediatric oncology. Note that separate chest CT (all indications) may be omitted if MRI clearly demonstrates pulmonary manifestations and if allowed by protocol (*if required by protocol or in the case of inconclusive MRI).

Table 5. Status quo DB 5: PET/MRI in paediatric oncology

Clinical evidence on the usefulness of PET/MRI in paediatric oncology Reduced radiation exposure as a key driver for PET/MRI of children

Initial results of a complementary role of advanced MR techniques for restaging of lymphoma patients

\begin{tabular}{ccc}
2012 & 2013 & 2014 \\
\hline$\leftrightarrow$ & $\nearrow$ & $\nearrow$ \\
$\nearrow$ & $\uparrow$ & $\uparrow$ \\
$\leftrightarrow$ & $\leftrightarrow$ & $\nearrow$
\end{tabular}


should be performed compared with MRI alone, and perhaps not surprisingly, in which SUV cut-off will provide the greatest accuracy for detecting MPNST.

Hirsch et al. reported an improved detection of small lung metastases with the use of respiratory-triggered thoracic MR as an alternative to chest CT [63]. Others have reported on the added value of a contrast-enhanced VIBE sequence for lung nodule detection [68]. Chest CT is still mandatory in both ongoing US and European paediatric HL treatment protocols, although in some cases, lymphoma patients undergoing a PET/MRI examination for response assessment may not need repeat CT imaging of the thorax as discussed previously. The panellists reported that the majority of patients (including children as young as 6 years of age) were able to undergo the examination without sedation, noting that child-specific patient preparation procedures, particularly in the outpatient setting, are essential.

All panellists provided compelling arguments for moving to PET/MRI in an effort to achieve radiation doses that aim for "As Low As Reasonably Achievable" (ALARA). It was shown that substantial reductions in effective patient radiation dose from $\sim 25 \mathrm{mSv}$ [67] for combined diagnostic $\mathrm{CT}$ and PET/CT exams to $\sim 7 \mathrm{mSv}$ for the PET/MRI examination can be achieved using the recommended activities for 3D acquisition of the European Association of Nuclear Medicine (EANM) guideline for $\left[{ }^{18} \mathrm{~F}\right] \mathrm{FDG}$-PET in paediatric oncology $[69,70]$.

\section{Future Challenges}

To date, there has been considerable variability in administered dose levels of $\left[{ }^{18} \mathrm{~F}\right] \mathrm{FDG}$, particularly with some PET/ MRI studies being performed after PET/CT as part of combined examinations. $\left[{ }^{18} \mathrm{~F}\right] \mathrm{FDG}$ administration should follow the EANM paediatric dosage card with the recommended weight-dependent standard activities for 3D PET acquisitions [71]. Discussions are ongoing regarding the higher $\left[{ }^{18} \mathrm{~F}\right] \mathrm{FDG}$ radioactivity levels given in the harmonisation project of 2014 [72]. However, in view of the increased PET(/MR) acquisition times $(\sim 7 \mathrm{~min} / \mathrm{bed}$ position), panellists noted an opportunity to further reduce the amount of injected activity by $30-50 \%$ of the weightdependent standard activity, leading to additional decreases in patient exposure without compromising image quality. This could result in effective doses for $\left[{ }^{18} \mathrm{~F}\right]$ FDG-PET/MRI in children well below $3 \mathrm{mSv}$.

The panel agreed on including whole-body DWI in their standard whole-body MR protocol as part of the combined PET/MRI study. There are limited data showing the added value of combining ADC measurements with quantitative $\left[{ }^{18} \mathrm{~F}\right] \mathrm{FDG}$ uptake. Standard MR-AC methods today do not adequately account for bone attenuation. Extending existing ultrashort TE (UTE) sequences to whole-body sequences will be important for paediatric oncology applications where bone marrow involvement is common, and bone attenuation can affect SUV quantification.

Non-oncologic investigations in children are still limited and include rheumatologic, infectious, neurologic and orthopaedic/sports medicine applications. The panel agreed that the future success of PET/MRI depends on expanding the applications to include both non-oncologic processes and other non- $\left[{ }^{18} \mathrm{~F}\right] \mathrm{FDG}-\mathrm{PET}$ tracers (e.g., $\left[{ }^{18} \mathrm{~F}\right] \mathrm{FDOPA}$, $\left[{ }^{68} \mathrm{Ga}\right]$ DOTATATE in neuroblastoma).

\section{Dialogue Board 6: PET/MRI in Cardiology}

\section{The Issues}

The adoption of combined PET/MRI for cardiology and cardiovascular disease (CVD) has just begun, and experiences are rather limited. Before PET/MRI can be used in clinical research, or even routine imaging, a number of methodological challenges regarding data acquisition, postprocessing and quantification have to be addressed. For example, standard MR-AC yields a bias in tissue radioactivity concentrations of up to $30 \%$, which may affect the differentiation of viable myocardium from scar tissue. Perhaps more importantly, substantial motion-induced misalignment affects the reliability of absolute quantification of myocardial perfusion imaging using $\left[{ }^{15} \mathrm{O}\right]$ water, $\left[{ }^{13} \mathrm{~N}\right] \mathrm{am}-$ monia and also $\left[{ }^{82} \mathrm{Rb}\right]$ rubidium chloride. While the simultaneous acquisition of dynamic PET and MRI data is, in principle, possible in both perfusion and viability protocols, the extraction of the PET list mode data for post-processing (e.g., rebinning, gating) is a major challenge in the absence of user-friendly data-handling tools. Finally, the integration and joint display of parametric images and other quantitative data is not yet easily facilitated with the user interfaces currently available. Likewise, fused dynamic sequences, whole-heart views, heart axis reorientation or multi-modal polar plot ("bull's eye") displays are missing in many general software packages, thus rendering the usefulness of combined PET/MRI low for clinical research applications today, unless major in-house programming supports subsequent data analysis workflows.

\section{Recent Advances or Achievements}

There is recent evidence of the ability of specific MRI protocols to deliver parameters that help identify unstable plaque structures in the context of atherosclerosis of the carotids. In addition, vascular inflammation, an important predicting factor for plaque instability, can be estimated reliably with $\left[{ }^{18} \mathrm{~F}\right] \mathrm{FDG}$ and also with $\left[{ }^{18} \mathrm{~F}\right]$ fluoride PET $[73$, 74], both offering the advantage of a whole-body approach that includes the assessment of large vessels (Fig. 4). A number of groups have already commenced feasibility studies of PET/MR for carotid imaging, where the 

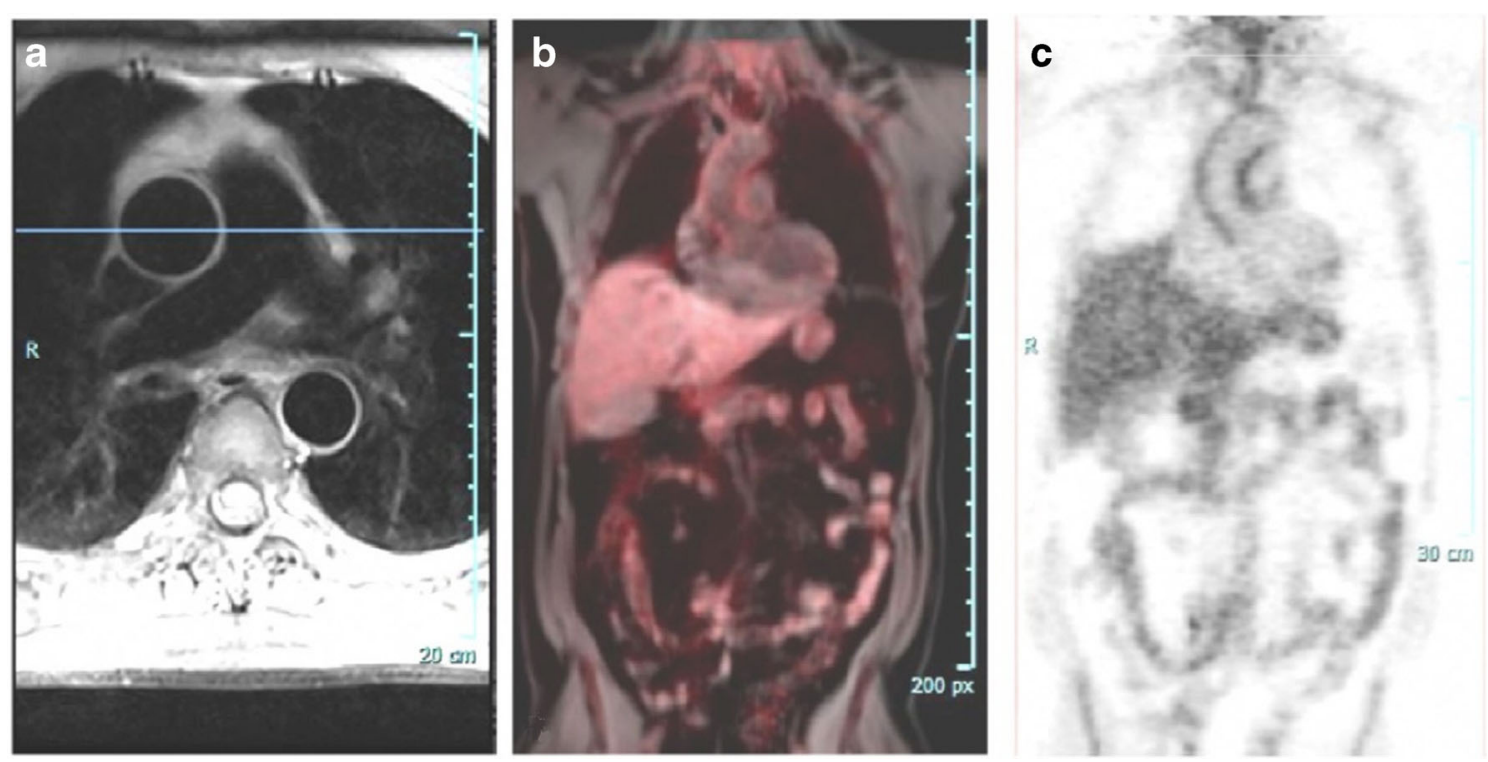

Fig. 4 An example of psoriatic arteritis imaged with simultaneous $\left[{ }^{18} \mathrm{~F}\right] \mathrm{FDG}-\mathrm{PET} / \mathrm{MRI}$ : a MRI, $\mathbf{b}$ fused PET and MR, and $\mathbf{c}$ PET.

attenuation correction of MR carotid coils potentially hampers the quantification of the PET signal, so that PET SUV would have to be adapted from published thresholds of the PET/CT systems.

\section{New Evidence That Has Been Reported (Table 6)}

Besides standard PET biomarkers for perfusion and viability estimation, there are further imaging biomarkers available for cardiovascular applications. Of special interest are biomarkers, which potentially add important information to the MRI parameters, such as innervation tracers ([ $\left.{ }^{11} \mathrm{C}\right]-(-)-m$-hydroxyephedrine (HED) and $N$-[3bromo-4-(3-[ $\left.{ }^{18} \mathrm{~F}\right]$ fluoro-propoxy)-benzyl]-guanidine (LMI1195)), tracers for the detection of infection and inflammation like sarcoidosis or tracers for the detection and differential diagnosis of structural heart disease (e.g., amyloid deposition, 2-(4'-[ $\left.{ }^{11} \mathrm{C}\right]$ methylaminophenyl)-6hydroxybenzothiazole (PIB) or novel fluorinated tracers). Also, chronic inflammation or fibrosis is of increasing interest, so that, for example, MRI T1w fibrosis mapping [75] in combination with neo-angiogenesis markers $\left(\left[{ }^{18} \mathrm{~F}\right]\right.$ fluoro-RGD) or collagen-specific markers [76] could improve the differential diagnosis of non-ischaemic cardiomyopathies.

\section{Future Challenges}

The panellists agreed on the need for analysis tools for standard and research applications of PET/MRI for cardiovascular disease. In parallel, users must identify the strongest parameters from each modality through fair comparison studies from simultaneous acquisitions in order to avoid redundant information from PET/MRI acquisitions. In parallel, protocols need to be adjusted and tailored to an overall examination time of $30 \mathrm{~min}$, or less for increased patient comfort. As with other applications of PET/MRI, protocol standardisation and harmonisation efforts must be followed.

\section{Round-table (RT1): Key Applications of PET/MRI}

\section{The Issues}

In concordance with the outcome of the second workshop [2], panellists and participants of this dialogue board agreed on the sole term "key application", rather than the more emotive and sensationalist "killer application", to describe a "a process for gathering new information that is substantially more useful and provides greater diagnostic clarity than any other measure at this time". Despite the agreement on this definition, the workshop attendees failed to nominate a

Table 6. Status quo DB 6: PET/MRI in cardiology

\begin{tabular}{|c|c|c|c|}
\hline & 2012 & 2013 & 2014 \\
\hline Resolution of methodological issues for CVD imaging (MR-AC, motion correction) & NA & $\nearrow$ & $\nearrow$ \\
\hline Develop analysis tools for standard CVD applications & NA & $\leftrightarrow$ & $\leftrightarrow$ \\
\hline Identification of key parameters/biomarkers from PET and MR to avoid redundancy in PET/MRI data & NA & $\leftrightarrow$ & $\nearrow$ \\
\hline Standardised imaging protocols & NA & $\leftrightarrow$ & $\leftrightarrow$ \\
\hline
\end{tabular}


single key application. As discussed in DB1, one reason may lie in the limited usefulness of $\left[{ }^{18} \mathrm{~F}\right] \mathrm{FDG}$ in key oncologic imaging applications of MR (and, thus, PET/MRI), such as imaging of brain tumours and local and metastatic prostate cancer.

Since the last workshop held a year ago, the rate of PET/ MRI system installations has not increased dramatically; this attests to the continued lack of clearly defined and accepted key applications. While several attendees voiced their preference for the use of PET/MRI for selected indications, such as paediatric imaging and imaging neuropsychiatric disorders, little evidence was available to validate the selection of these applications from multiple centres. This lack of perceived significant progress can perhaps be explained by the current focus on clinical indications that are well served already by MR-only or combined PET/CT imaging, namely, cardiovascular disease and cancer. While cancer is perceived by many to represent the largest health care burden, other diseases such as diabetes, joint disorders or mental illnesses cause similar health care burdens and productivity losses as cancer and potentially may be more promising targets for a new imaging modality such as PET/ MRI. Other applications of PET/MRI in the future may include imaging of advanced coronary artery disease and heart failure and imaging of infection and inflammation.

PET/MRI does hold the potential to provide a number of methodological improvements over separate PET and MR imaging and over PET/CT imaging. These include, but are not limited to, accurate patient alignment, the recording of dynamic and moving phenomena, an absolute match between tissue information from both modalities under identical physiological conditions, and better localisation of PET signal in the soft tissue. In spite of more research teams making greater efforts to assess these potential improvements, no major advance has been reported yet for clinical routine use.

Expanding on the clinical scope, workshop attendees discussed the need to standardise PET/MRI imaging protocols as a prerequisite for supporting PET/MRI for selected key applications. While PET and PET/CT standardisation are comparatively well advanced and accepted among nuclear medicine specialists, MR standardisation remains problematic. With PET/MRI, in particular, imaging protocols can become "open-ended" as more, often superfluous, MR sequences are added to the examination. This observation has prompted further discussions aimed at reducing the overall examination time to $30 \mathrm{~min}$ or less for oncology indications.

The perceived complexity of PET/MRI protocols (as discussed already in [2]) has led attendees to question the scope of a key application of PET/MRI. Subsequently, several panellists and audience members suggested that "research" was actually the key application of PET/MRI today. All agreed that for a key clinical application of PET/MRI to be found, four major hurdles need to be addressed:

- The number of approved and reimbursed PET tracers must be increased.

- PET/MRI acquisition protocols must be standardised with total examination times being tolerable for patients.

- Significant improvements in acquisition and analysis software matching the state-of-the-art in other imaging technologies, such as stand-alone MRI and PET/CT.

- Clinical PET/MRI reports must be fully integrated.

\section{Recent Advances or Achievements}

Perhaps the most positive statement made during this workshop was "Clinical PET/MRI is now feasible". This comment reflects the increasing number of studies performed, even with full awareness of the limitations of combined PET/MRI today, such as biased, standard MR-AC and a number of imaging artefacts specific to PET/MRI. Methodological improvements were discussed in DB2 and include advances in the reproducibility of MR-based attenuation coefficients. As in 2013, workshop attendees agreed on paediatric oncology, brain tumour imaging and neurodegenerative diseases being worthy indications for combined PET/MRI. However, few (if any) additional clinical data from prospective studies have become available to support this consensus.

\section{New Evidence That Has Been Reported (Table 7)}

The panel agreed that there is growing evidence supporting the clinical usefulness of PET/MRI in paediatric oncology and brain imaging.

Table 7. Status quo RT1: Key applications for PET/MRI

\begin{tabular}{lccc}
\hline & 2012 & 2013 & 2014 \\
\cline { 2 - 3 } Paediatric oncology is to become a key application of PET/MRI & $\nearrow$ & $\nearrow$ \\
Dementia is to become a key application of PET/MRI & $\nearrow$ & $\uparrow$ & $\nearrow$ \\
Neuro-oncology is to become a key application of PET/MRI & $\nearrow$ & $\leftrightarrow$ \\
Cardiovascular imaging is to become a key application of PET/MRI & $\leftrightarrow$ & $\nearrow$ \\
Multi-centre evaluation of clinical PET/MRI & $\uparrow$ & $\nearrow$ \\
Multi-parametric imaging is a key driver for PET/MRI & $\leftrightarrow$ & $\nearrow$ \\
\hline
\end{tabular}




\section{Future Challenges}

The attendees agreed on three main challenges to successful clinical adoption of PET/MR. First, imaging protocols need to be standardised, limited in time and limited to the essential MR sequences for a given clinical indication. Second, the combination of PET and MR needs to be mirrored by a cooperative effort of complementary medical and technical disciplines. As a first step, PET/MRI reports should be fully integrated. Third, the commercial availability of PET tracers, interrogating new aspects of tumour biology, should be expanded.

In summary, a few PET/MRI actions were proposed:

- Shorten clinical PET/MRI examination times

- Standardise PET/MRI protocols within a given centre

- Harmonise PET/MRI protocols across sites

- Prepare for collecting multi-centre evidence

- Consider a (paediatric) PET/MRI registry

Finally, one of the speakers appeared to sum up the mood of the entire meeting in commenting that, "the real work has just begun" with PET/MRI, and exciting times and challenges lie ahead.

\section{Summary}

Combined PET/MR imaging was proposed for imaging patients in the mid-2000s, and the first prototype designs became available at selected sites in 2006 [77, 78]. Since then, tremendous progress has been made with regard to technical versatility and methodological approaches. Today, three major imaging hardware vendors offer PET/MRI with varying system designs. Most notably, more fully integrated PET/MRI systems than co-planar PET/MRI systems have been installed. Of the systems installed worldwide, about one fifth have a co-planar PET/MRI system. Interestingly, the clinical evidence of the need to perform PET and MR imaging simultaneously rather than with a minimal offset in time is limited. However, recent advances in clinical research with PET/MRI favour the simultaneous acquisition, as discussed in "Dialogue Board 4: Advanced PET/ MRI-Multi-Parametric Imaging" in particular.

General expectations for PET/MRI remain high. One author/panellist (CLF) proposed the picture of an "egglaying-wool-milk-sow" (a terminology translated from its German origin-Eierlegende Wollmilchsau), to describe an animal that provides all kinds of food supplies and materials at once. It became clear from this workshop that PET/MRI is a long way from providing all types of information at once, especially in a given and acceptable time. Perhaps most importantly, this workshop suggested that consensus on what information and what range of parameters is desired was lacking for many applications of PET/MRI. Such consensus, driven by a particular application or research interest, is needed to support the establishment of PET/MRI in clinical routine.

Workshop panellists agreed that lessons should be learned from the adoption of PET/CT and that PET/MRI needs to be accepted as a single modality from the start. This unified recognition will aid the adoption of PET/ MRI in a clinical environment of steadily growing complexity. Likewise, the future of PET/MRI was seen not in being used by an individual medical specialist but rather in providing multi-parametric data that should be interpreted in a multi-disciplinary approach. PET/MRI, whilst offering an integrated technical platform, also requires an integrated approach from complementary medical specialists. The need to explore data mining of already acquired (multi-centre) data, as well as of multiparametric data to be acquired, was recognised as a key challenge for PET/MRI users. Finally, this workshop concluded that technical innovation with PET/MRI outpaces regulatory processes and legislation that are essential for the local adoption and wider dissemination of this imaging technology in modern health care systems.

Recognising the outcome of the discussions of the previous two workshops held in Tübingen in 2012 and $2013[1,2]$, panellists at this year's workshop were able to further specify progress made in the areas of oncology, cardiology, neurology and paediatric imaging as well as in methodology and quantification and multi-parametric imaging. We have included our summary perspectives in the status quo tables following the descriptions of each dialogue board.

This workshop identified four main challenges that must be addressed to speed up the adoption of PET/MRI in clinical practice.

1. The total examination time needs to be less than $30 \mathrm{~min}$.

2. PET/MRI imaging protocols must be standardised.

3. To allow full quantification of the PET image data, full inter-vendor comparability of MR-based attenuation values must be ensured.

4. Explore a holistic approach to multi-parametric imaging and data mining

It was the intent of all panellists to continue the course of this workshop, and therefore, the next workshop will be held in Tübingen from February 23 to 27, 2015. By then, workshop attendees will need to review progress on the following steps identified at this year's meeting as necessary short-term activities for bolstering the promise of combined and integrated PET/MRI: (i) set up a central PET/MRI registry, (ii) establish common quality control guidelines for PET/MR and (iii) develop analysis tools that provide quantitative image information beyond simple SUV analysis.

As stated in the title of this workshop review: "the real work has just started". Let us take this as a positive spin on PET/MRI and join forces. 
Acknowledgments. We wish to thank all participants for their active contribution at the third Tübingen Workshop on PET/MRI and for the lively discussions.

The workshop was endorsed by the following societies: European Cooperation in Science and Technology (COST), European Association of Nuclear Medicine (EANM), European Society of Magnetic Resonance in Medicine and Biology (ESMRMB), European Society of Molecular Imaging (ESMI), German Society of Nuclear Medicine (DGN), German Society of Radiology (DGR), Society of Nuclear Medicine and Molecular Imaging (SNMMI), and World Molecular Imaging Society (WMIS).

We would further like to acknowledge the generous support of the workshop sponsors: Bayer HealthCare, GE Healthcare, Hermes Medical Solutions, Mediso Medical Imaging Systems, Mirada Medical, П.pmod Biomedical Image Quantification, Siemens Healthcare and the WMIS.

Conflict of Interest. The authors declare that in respect of the preparation and content of this manuscript, they have no conflict of interest.

\section{References}

1. Bailey DL, Barthel H, Beyer T et al (2013) Summary report of the first international workshop on PET/MR imaging, march 19-23, 2012, Tubingen, Germany. Mol Imaging Biol MIB Off Publ Acad Mol Imaging 15:361-371

2. Bailey DL, Barthel H, Beuthin-Baumann B et al (2014) Combined PET/MR: where are we now? Summary report of the second international workshop on PET/MR imaging april 8-12, 2013, Tubingen, Germany. Mol Imaging Biol MIB Off Publ Acad Mol Imaging 16:295-310

3. Kubiessa K, Purz S, Gawlitza M et al (2014) Initial clinical results of simultaneous ${ }^{18} \mathrm{~F}$-FDG PET/MRI in comparison to ${ }^{18} \mathrm{~F}$-FDG PET/CT in patients with head and neck cancer. Eur J Nucl Med Mol Imaging 41:639-648

4. Lee G, Kim IH, Kim SJ et al (2014) Clinical implication of PET/MR imaging in preoperative esophageal cancer staging: comparison with PET/CT, endoscopic ultrasonography, and CT. J Nucl Med Off Publ Soc Nucl Med 55:1242-1247

5. Beiderwellen KJ, Poeppel TD, Hartung-Knemeyer V et al (2013) Simultaneous ${ }^{68} \mathrm{Ga}$-DOTATOC PET/MRI in patients with gastroenteropancreatic neuroendocrine tumors: initial results. Invest Radiol 48:273-279

6. Hillner BE, Liu D, Coleman RE et al (2007) The national oncologic PET registry (NOPR): design and analysis plan. J Nucl Med Off Publ Soc Nucl Med 48:1901-1908

7. Hillner BE, Siegel BA, Hanna L et al (2012) Impact of ${ }^{18}$ F-FDG PET used after initial treatment of cancer: comparison of the National Oncologic PET Registry 2006 and 2009 cohorts. J Nucl Med Off Publ Soc Nucl Med 53:831-837

8. Hillner BE, Siegel BA, Liu D et al (2008) Impact of positron emission tomography/computed tomography and positron emission tomography (PET) alone on expected management of patients with cancer: initial results from the national oncologic PET registry. J Clin Oncol Off J Am Soc Clin Oncol 26:2155-2161

9. Hillner BE, Siegel BA, Shields AF et al (2009) The impact of positron emission tomography (PET) on expected management during cancer treatment: findings of the national oncologic PET registry. Cancer $115: 410-418$

10. Aznar MC, Sersar R, Saabye J et al (2014) Whole-body PET/MRI: the effect of bone attenuation during MR-based attenuation correction in oncology imaging. Eur J Radiol 83:1177-1183

11. Bezrukov I, Schmidt H, Mantlik F et al (2013) MR-based attenuation correction methods for improved PET quantification in lesions within bone and susceptibility artifact regions. J Nucl Med Off Publ Soc Nucl Med 54:1768-1774

12. Kuhn FP, Hullner M, Mader CE et al (2014) Contrast-enhanced PET/ MR imaging versus contrast-enhanced PET/CT in head and neck cancer: how much MR information is needed? J Nucl Med Off Publ Soc Nucl Med 55:551-558

13. Pace L, Nicolai E, Luongo A et al (2014) Comparison of whole-body PET/CT and PET/MRI in breast cancer patients: lesion detection and quantitation of ${ }^{18} \mathrm{~F}$-deoxyglucose uptake in lesions and in normal organ tissues. Eur J Radiol 83:289-296
14. Preuss M, Werner P, Barthel $\mathrm{H}$ et al (2014) Integrated PET/MRI for planning navigated biopsies in pediatric brain tumors. Child's Nerv Syst ChNS Off J Int Soc Pediatr Neurosurg 30:1399-1403

15. Purz S, Sabri O, Viehweger A et al (2014) Potential pediatric applications of PET/MR. J Nucl Med Off Publ Soc Nucl Med 55:32S-39S

16. Schwenzer NF, Schmidt H, Gatidis S, et al. (2013) Measurement of apparent diffusion coefficient with simultaneous MR/positron emission tomography in patients with peritoneal carcinomatosis: comparison with ${ }^{18}$ F-FDG-PET. JMRI-J Magn Reson Im

17. Bisdas S, Ritz R, Bender B et al (2013) Metabolic mapping of gliomas using hybrid MR-PET imaging: feasibility of the method and spatial distribution of metabolic changes. Invest Radiol 48:295301

18. Wetter A (2014) Molecular research in urology 2014: update on PET/ MR imaging of the prostate. Int J Mol Sci 15:13401-13405

19. Wetter A, Lipponer C, Nensa F et al (2014) Evaluation of the PET component of simultaneous [(18) F] choline PET/MRI in prostate cancer: comparison with [(18) F] choline PET/CT. Eur J Nucl Med Mol Imaging 41:79-88

20. Wetter A, Lipponer C, Nensa F et al (2014) Quantitative evaluation of bone metastases from prostate cancer with simultaneous $\left[{ }^{18} \mathrm{~F}\right]$ choline PET/MRI: combined SUV and ADC analysis. Ann Nucl Med 28:405410

21. Wetter A, Nensa F, Schenck M et al (2014) Combined PET imaging and diffusion-weighted imaging of intermediate and high-risk primary prostate carcinomas with simultaneous $\left[{ }^{18} \mathrm{~F}\right]$ choline PET/MRI. PLoS One 9:e101571

22. Afshar-Oromieh A, Haberkorn U, Schlemmer HP et al (2014) Comparison of PET/CT and PET/MRI hybrid systems using a ${ }^{68} \mathrm{Ga}-$ labelled PSMA ligand for the diagnosis of recurrent prostate cancer: initial experience. Eur J Nucl Med Mol Imaging 41:887-897

23. Huellner MW, Appenzeller P, Kuhn FP, et al (2014) Whole-body nonenhanced PET/MR versus PET/CT in the staging and restaging of cancers: preliminary observations. Radiology: 140090

24. Martinez-Moller A, Souvatzoglou M, Delso G et al (2009) Tissue classification as a potential approach for attenuation correction in whole-body PET/MRI: evaluation with PET/CT data. J Nucl Med Off Publ Soc Nucl Med 50:520-526

25. Schramm G, Langner J, Hofheinz F et al (2013) Quantitative accuracy of attenuation correction in the Philips ingenuity TF whole-body PET/ MR system: a direct comparison with transmission-based attenuation correction. MAGMA 26:115-126

26. Prieto E, Dominguez-Prado I, Garcia-Velloso MJ et al (2013) Impact of time-of-flight and point-spread-function in SUV quantification for oncological PET. Clin Nucl Med 38:103-109

27. Nuyts J, Bal G, Kehren F et al (2013) Completion of a truncated attenuation image from the attenuated PET emission data. IEEE Trans Med Imaging 32:237-246

28. Nuyts J, Dupont P, Stroobants S et al (1999) Simultaneous maximum a posteriori reconstruction of attenuation and activity distributions from emission sinograms. IEEE Trans Med Imaging 18:393-403

29. Rezaei A, Defrise M, Bal G et al (2012) Simultaneous reconstruction of activity and attenuation in time-of-flight PET. IEEE Trans Med Imaging 31:2224-2233

30. Rezaei A, Defrise M, Nuyts J (2014) ML-reconstruction for TOF-PET with simultaneous estimation of the attenuation factors. IEEE Trans Med Imaging 33:1563-1572

31. Blumhagen JO, Braun H, Ladebeck R et al (2014) Field of view extension and truncation correction for MR-based human attenuation correction in simultaneous MR/PET imaging. Med Phys 41:022303

32. Kartmann R, Paulus DH, Braun H et al (2013) Integrated PET/MR imaging: automatic attenuation correction of flexible RF coils. Med Phys 40:082301

33. Defrise M, Rezaei A, Nuyts J (2012) Time-of-flight PET data determine the attenuation sinogram up to a constant. Phys Med Biol $57: 885-899$

34. Defrise M, Rezaei A, Nuyts J (2014) Transmission-less attenuation correction in time-of-flight PET: analysis of a discrete iterative algorithm. Phys Med Biol 59:1073-1095

35. Wurslin C, Schmidt H, Martirosian P et al (2013) Respiratory motion correction in oncologic PET using T1-weighted MR imaging on a simultaneous whole-body PET/MR system. J Nucl Med Off Publ Soc Nucl Med 54:464-471 
36. Lougovski A, Hofheinz F, Maus J et al (2014) A volume of intersection approach for on-the-fly system matrix calculation in 3D PET image reconstruction. Phys Med Biol 59:561-577

37. Weber WA, Ziegler SI, Thodtmann R et al (1999) Reproducibility of metabolic measurements in malignant tumors using FDG PET. J Nucl Med Off Publ Soc Nucl Med 40:1771-1777

38. Ziegler S, Braun H, Ritt P et al (2013) Systematic evaluation of phantom fluids for simultaneous PET/MR hybrid imaging. J Nucl Med Off Publ Soc Nucl Med 54:1464-1471

39. Oehmigen M, Ziegler S, Jakoby BW et al (2014) Radiotracer dose reduction in integrated PET/MR: implications from National Electrical Manufacturers Association phantom studies. J Nucl Med Off Publ Soc Nucl Med 55:1361-1367

40. van den Hoff J, Oehme L, Schramm G et al (2013) The PET-derived tumorto-blood standard uptake ratio (SUR) is superior to tumor SUV as a surrogate parameter of the metabolic rate of FDG. EJNMMI Res 3:77

41. van den Hoff J, Lougovski A, Schramm G et al (2014) Correction of scan time dependence of standard uptake values in oncological PET. EJNMMI Res 4:18

42. Heiss WD (2009) The potential of PET/MR for brain imaging. Eur J Nucl Med Mol Imaging 36(Suppl 1):S105-S112

43. Catana C, Drzezga A, Heiss WD, Rosen BR (2012) PET/MRI for neurologic applications. J Nucl Med Off Publ Soc Nucl Med 53:1916-1925

44. Drzezga A, Barthel H, Minoshima S, Sabri O (2014) Potential clinical applications of PET/MR imaging in neurodegenerative diseases. J Nucl Med Off Publ Soc Nucl Med 55:47S-55S

45. Garibotto V, Heinzer S, Vulliemoz S et al (2013) Clinical applications of hybrid PET/MRI in neuroimaging. Clin Nucl Med 38:13-18

46. Riedl V, Bienkowska K, Strobel C et al (2014) Local activity determines functional connectivity in the resting human brain: a simultaneous FDG-PET/fMRI study. J Neurosci Off J Soc Neurosci 34:6260-6266

47. Hitz S, Habekost C, Fürst S et al (2014) Systematic comparison of the performance of integrated whole-body PET/MR imaging to conventional PET/CT for ${ }^{18} \mathrm{~F}-\mathrm{FDG}$ brain imaging in patients examined for suspected dementia. J Nucl Med Off Publ Soc Nucl Med 55:923-931

48. Andersen FL, Ladefoged CN, Beyer T et al (2014) Combined PET/MR imaging in neurology: MR-based attenuation correction implies a strong spatial bias when ignoring bone. Neuroimage 84:206-216

49. Poynton CB, Chen KT, Chonde DB et al (2014) Probabilistic atlasbased segmentation of combined T1-weighted and DUTE MRI for calculation of head attenuation maps in integrated PET/MRI scanners Am J Nucl Med Mol Imaging 4:160-171

50. Stegger L, Martirosian P, Schwenzer N et al (2012) Simultaneous PET/ MR imaging of the brain: feasibility of cerebral blood flow measurements with FAIR-TrueFISP arterial spin labeling MRI. Acta Radiol 53:1066-1072

51. Werner P, Fritzsch D, Holland H et al (2014) Definition of primary and secondary glioblastoma-letter. Clin Cancer Res An Off J Am Assoc Cancer Res 20:2011-2012

52. Panagiotidis E, Shankar A, Afaq A, Bomanji J (2014) Assessing therapy response of secreting pineal germ cell tumor on simultaneous ${ }^{18}$ F-choline PET/MRI. Clin Nucl Med 39:e387-e388

53. Sander CY, Hooker JM, Catana C et al (2013) Neurovascular coupling to D2/D3 dopamine receptor occupancy using simultaneous PET/ functional MRI. Proc Natl Acad Sci U S A 110:11169-11174

54. Kendziorra K, Wolf H, Meyer PM et al (2011) Decreased cerebral alpha4beta2* nicotinic acetylcholine receptor availability in patients with mild cognitive impairment and Alzheimer's disease assessed with positron emission tomography. Eur J Nucl Med Mol Imaging 38:515-525

55. Deutschlander A, Stephan T, Riedel E et al (2008) Nicotine-induced nystagmus correlates with midpontine activation. Neuroimage 41:479-482

56. Mitsias PD, Ewing JR, Lu M et al (2004) Multiparametric iterative selforganizing MR imaging data analysis technique for assessment of tissue viability in acute cerebral ischemia. AJNR Am J Neuroradiol 25:14991508

57. Langer DL, van der Kwast TH, Evans AJ et al (2009) Prostate cancer detection with multi-parametric MRI: logistic regression analysis of quantitative T2, diffusion-weighted imaging, and dynamic contrastenhanced MRI. J Magn Reson Imaging 30:327-334
58. Liu X, Langer DL, Haider MA et al (2009) Prostate cancer segmentation with simultaneous estimation of Markov random field parameters and class. IEEE Trans Med Imaging 28:906-915

59. Wehrl HF, Schwab J, Hasenbach K et al (2013) Multimodal elucidation of choline metabolism in a murine glioma model using magnetic resonance spectroscopy and ${ }^{11} \mathrm{C}$-choline positron emission tomography. Cancer Res 73:1470-1480

60. Wehrl HF, Hossain M, Lankes K et al (2013) Simultaneous PET-MRI reveals brain function in activated and resting state on metabolic, hemodynamic and multiple temporal scales. Nat Med 19:1184-1189

61. Schmidt H, Brendle C, Schraml C et al (2013) Correlation of simultaneously acquired diffusion-weighted imaging and 2-deoxy$\left[{ }^{18} \mathrm{~F}\right]$ fluoro-2-D-glucose positron emission tomography of pulmonary lesions in a dedicated whole-body magnetic resonance/positron emission tomography system. Invest Radiol 48:247-255

62. Dukart J, Mueller K, Horstmann A et al (2011) Combined evaluation of FDG-PET and MRI improves detection and differentiation of dementia. PLoS One 6:e18111

63. Hirsch FW, Sattler B, Sorge I et al (2013) PET/MR in children. Initial clinical experience in paediatric oncology using an integrated PET/MR scanner. Pediatr Radiol 43:860-875

64. Punwani S, Cheung KK, Skipper N et al (2013) Dynamic contrastenhanced MRI improves accuracy for detecting focal splenic involvement in children and adolescents with Hodgkin disease. Pediatr Radiol 43:941-949

65. Punwani S, Taylor SA, Saad ZZ et al (2013) Diffusion-weighted MRI of lymphoma: prognostic utility and implications for PET/MRI? Eur J Nucl Med Mol Imaging 40:373-385

66. Inaoka T, Takahashi K, Mineta M et al (2007) Thymic hyperplasia and thymus gland tumors: differentiation with chemical shift MR imaging. Radiology 243:869-876

67. Chawla SC, Federman N, Zhang D et al (2010) Estimated cumulative radiation dose from PET/CT in children with malignancies: a 5-year retrospective review. Pediatr Radiol 40:681-686

68. Rauscher I, Eiber M, Furst S et al (2014) PET/MR imaging in the detection and characterization of pulmonary lesions: technical and diagnostic evaluation in comparison to PET/CT. J Nucl Med Off Publ Soc Nucl Med 55:724-729

69. Stauss J, Franzius C, Pfluger $T$ et al (2008) Guidelines for ${ }^{18}$ F-FDG PET and PET-CT imaging in paediatric oncology. Eur J Nucl Med Mol Imaging 35:1581-1588

70. Schafer JF, Gatidis S, Schmidt H et al (2014) Simultaneous whole-body PET/MR imaging in comparison to PET/CT in pediatric oncology: initial results. Radiology 273:220-231

71. Lassmann M, Biassoni L, Monsieurs M et al (2008) The new EANM paediatric dosage card: additional notes with respect to F-18. Eur J Nucl Med Mol Imaging 35:1666-1668

72. Lassmann M, Treves ST (2014) Pediatric Radiopharmaceutical Administration: harmonization of the 2007 EANM Paediatric Dosage Card (Version 1.5.2008) and the (2010) North american consensus guideline. Eur J Nucl Med Mol Imaging 41:1636

73. Dweck MR, Chow MW, Joshi NV et al (2012) Coronary arterial ${ }^{18} \mathrm{~F}-$ sodium fluoride uptake: a novel marker of plaque biology. J Am Coll Cardiol 59:1539-1548

74. Joshi NV, Vesey AT, Williams MC et al (2014) ${ }^{18} \mathrm{~F}$-fluoride positron emission tomography for identification of ruptured and high-risk coronary atherosclerotic plaques: a prospective clinical trial. Lancet 383:705-713

75. Mewton N, Liu CY, Croisille P et al (2011) Assessment of myocardial fibrosis with cardiovascular magnetic resonance. J Am Coll Cardiol 57:891-903

76. Muzard J, Sarda-Mantel L, Loyau S et al (2009) Non-invasive molecular imaging of fibrosis using a collagen-targeted peptidomimetic of the platelet collagen receptor glycoprotein VI. PLoS One 4:e5585

77. Schlemmer HP, Pichler BJ, Schmand M et al (2008) Simultaneous MR/ PET imaging of the human brain: feasibility study. Radiology 248:1028-1035

78. Schmand M, Burbar Z, Corbeil JL et al (2007) BrainPET: first human tomograph for simultaneous (functional) PET and MR Imaging. J Nucl Med Off Publ Soc Nucl Med 48:45P 